\title{
Web Log Analysis Panel
}

\section{Bernard J. Jansen (moderator)}

School of Information Sciences and Technology, The Pennsylvania State

University, State College, PA 16803 jjansen@ist.psu.edu

Suresh Bhavnani (panelist)

School of Information, University of Michigan, Ann Arbor, MI 48109

bhavnani@umich.edu

Craig Murray (panelist)

College of Information Studies, University of Maryland, College Park, MD 20740 gcraigm@umd.edu

\section{Amanda Spink (panelist)}

Faculty of Information Technology, Queensland University of Technology, Brisbane QLD 4000 Australia ah.spink@qut.edu.au

\section{Dietmar Wolfram (panelist)}

School of Information Studies, University of Wisconsin-Milwaukee, Milwaukee, WI 53201 dwolfram@uwm.edu

A significant number of Web systems, search engines, and Websites collect large amount of user data in transaction and search logs. The availability of such logs has produced a wealth of research and development over the past decade on search patterns for Web search engines, intranet search services, target advertising, and Website design. These Web logs can certainly be rich sources of both personal data and user behavior. The availability of such data has resulted in significant advances in personalization, targeted search, and information push. However, these logs provide challenges and risks, along with opportunities. Panel members will discuss which critical research challenges need to be addressed in the near future. The panel members will also engage in a dialogue with audience members to develop an agenda for advancing the state of Web transaction and search log research on collecting Web log data, sharing data, addressing user privacy, analysis tools, and techniques to aid in Web log analysis. The panel and 
discussion will addresses aspects of log analysis focusing the users, metrics, technology, user - system interaction, policy, and balancing research objectives with privacy. Issues to be covered include (but are not limited to):

1. Security:

Can we design and implement standards for collecting log data and sharing logs?

2. Social:

What are the ethical issues of log analysis? What are the legal issues of log collection and use? Is there a social responsible for collecting and preserving log data, in addition to protecting privacy?

3. Standards:

Different researchers and practitioners define search process events differently. How can we implement standards in the log analysis area?

4. Techniques: What are effective and efficient methods and tools for log collection and analysis?

5. Technology:

Much log analysis results have focused on descriptive measures. How can findings best be translated into practice, whether for system design or service development? 